\title{
Laser pulse trains for controlling excited state dynamics of adenine in water
}

\author{
Jens Petersen, ${ }^{a}$ Matthias Wohlgemuth, ${ }^{a}$ Bernhard Sellner, ${ }^{b}$ Vlasta Bonačić-Koutecký, ${ }^{c, d}$ Hans \\ Lischka, ${ }^{b, e}$ and Roland Mitrić ${ }^{* a}$
}

\author{
Received Xth XXXXXXXXXX 20XX, Accepted Xth XXXXXXXXX 20XX \\ First published on the web $X t h X X X X X X X X X X 200 X$ \\ DOI: 10.1039/b000000x
}

We investigate theoretically the control of the ultrafast excited state dynamics of adenine in water by laser pulse trains, with the aim to extend the excited state lifetime and to suppress nonradiative relaxation processes. For this purpose, we introduce the combination of our field-induced surface hopping method (FISH) with the quantum mechanical-molecular mechanical (QM/MM) technique for simulating the laser-driven dynamics in the condensed phase under explicit inclusion of the solvent environment. Moreover, we employ parametric pulse shaping in the frequency domain in order to design simplified laser pulse trains allowing to establish a direct link between the pulse parameters and the controlled dynamics. We construct pulse trains which achieve a high excitation efficiency and at the same time keep a high excited state population for a significantly extended time period compared to the uncontrolled dynamics. The control mechanism involves a sequential cycling of the population between the lowest and higher excited states, thereby utilizing the properties of the corresponding potential energy surfaces to avoid conical intersections and thus to suppress the nonradiative decay to the ground state. Our findings provide a means to increase the fluorescence yield of molecules with an intrinsically very short excited state lifetime, which can lead to novel applications of shaped laser fields in the context of biosensing.

\section{Introduction}

The ultrafast dynamics of molecules is characterized by the interplay of light-induced excitations with the intrinsic nonadiabatic (vibronic) coupling of electronic states that both lead to time-dependent transitions between these states, ultimately determining the course of various fundamental photochemical processes. Stimulated by the progress in theory ${ }^{1-5}$, the experimental laser pulse shaping technology developed within the last decades ${ }^{6}$ has opened the route to actively manipulate various photochemical and photophysical processes on the time scale of nuclear ${ }^{7-10}$ and electronic motion ${ }^{11-14}$ and to control the outcome and yield of chemical reactions ${ }^{15-17}$. Such control is based on the optimization of the time, phase, frequency and polarization of the laser fields which allows to coherently drive the system and enhance the desired reaction product. The most widely used control strategy relies on the iterative optimization of the control field in the closed-loop learning scheme utilizing the response of the molecular system as feed-

\footnotetext{
a Freie Universität Berlin, Fachbereich Physik, Arnimallee 14, D-14195 Berlin, Germany; E-mail: mitric@zedat.fu-berlin.de,${ }^{b}$ Universität Wien, Institut für Theoretische Chemie, Währinger Str. 17, A-1090 Vienna, Austria, ${ }^{c}$ Humboldt-Universität zu Berlin, Institut für Chemie, Brook-Taylor-Straße 2, D-12489 Berlin, Germany, ${ }^{d}$ Interdisciplinary Center for Advanced Science and Technology, University of Split, Meštrovićevo Šetalište bb., HR-21000 Split, Croatia, ${ }^{e}$ Texas Tech University, Department of Chemistry \& Biochemistry, Memorial Circle \& Boston, Lubbock, TX 79409-1061, USA
}

back signal ${ }^{18}$. This method has been successfully applied to control processes like molecular fragmentation ${ }^{19,20}$, ionization $^{21-23}$ or isomerization ${ }^{24}$. However, in this way complex pulse forms are usually obtained and their interpretation is often far from being obvious. Therefore, solely experimental optimal control pulses are not sufficient to provide the mechanism behind the control and input from theory is highly desirable $22,23,25$. A particularly convenient strategy to achieve more interpretable results and to disentangle competing control pathways consists in the construction of simplified shaped laser pulses with only a small number of parameters which can be varied ${ }^{26-28}$. In this way, the effect of individual parameters can be easily deduced and efficient control fields can be obtained without performing a full iterative optimization procedure.

The theoretical treatment of coupled electron-nuclear dynamics driven by complex shaped laser pulses requires efficient methods for the propagation of nuclei as well as for the electronic structure including ground and excited electronic states. In this context, a particularly attractive approach which is generally applicable also to complex systems is based on the semiclassical molecular dynamics "on the fly", in which the nuclear motion is described by classical trajectories and the electronic structure is calculated using various quantum chemical methods. For the field-free nonadiabatic dynamics a variety of approaches has been devised, the most widely 
used being Tully's surface hopping procedure ${ }^{29,30}$. In contrast, the inclusion of laser fields into the molecular dynamics simulations has been only recently achieved in the framework of our field-induced surface hopping method (FISH) ${ }^{31}$. This approach has for the first time allowed to reveal the control mechanism responsible for the optimal dynamic discrimination of two almost indistinguishable flavin chromophores ${ }^{25}$. The particular advantage of the FISH method is that the experimentally shaped laser pulses can be directly applied in the theoretical simulation and the control mechanism can be immediately extracted. In addition, due to the classical treatment of the nuclear motion fairly large systems can be treated without the need to reduce the number of degrees of freedom as it is usually done in full quantum dynamics simulations. Moreover, since the application of optimally shaped pulses is particularly interesting in the context of the control of chemical processes in the condensed phase ${ }^{32}$, the FISH method combined with quantum mechanical/molecular mechanical (QM/MM) ${ }^{33-36}$ techniques offers a very attractive possibility to treat the dynamics of such complex systems.

Therefore, in this contribution we introduce the combination of FISH with QM/MM for explicit treatment of the solvent. Furthermore, the question can be raised if laser pulses can be designed in order to manipulate the excited state dynamics in molecules that exhibit ultrafast nonradiative relaxation, such as DNA nucleobases. This has been motivated by the idea to elongate the intrinsically very short excited state lifetimes and thus to enable for example selective spectroscopic studies of excited states or even to invoke fluorescence. As a prototype molecule, we have chosen the DNA base adenine in water environment. The mechanism of the ultrafast excited state decay of adenine has been the subject of numerous experimental and theoretical studies. In solution, the two tautomeric forms $7 \mathrm{H}$ - and $9 \mathrm{H}$-adenine are present, of which the $9 \mathrm{H}$-form is the dominant one ${ }^{37}$. Time-resolved photoelectron spectroscopy studies have shown that the excited state lifetime of the $9 \mathrm{H}$-tautomer in the gas phase after resonant excitation to the bright $\pi \pi^{*}$ states at $267 \mathrm{~nm}$ is about $750 \mathrm{fs}^{38}$. However, the lifetime is found to be strongly wavelength dependent, reaching up to $9 \mathrm{ps}$ for excitation at $277 \mathrm{~nm}^{39}$. Theoretical studies based on nonadiabatic molecular dynamics using both semiempirical $^{40}$ and ab initio quantum chemical methods ${ }^{41}$ have revealed a two-step relaxation mechanism involving different conical intersections between the electronic states. The initially excited bright state relaxes very rapidly to the lowest excited state with a time constant of $\sim 20 \mathrm{fs}$, followed by the relaxation to the ground state with a time constant of $\sim 550$ fs.

In aqueous solution the time scales for nonradiative relaxation are considerably shorter. Experimental investigations have obtained lifetimes for the dominant $9 \mathrm{H}$-tautomer of 180 fs by transient absorption ${ }^{37}$, about 550 fs by time-resolved fluorescence $^{42}$ and $<300$ fs by photon echo experiments ${ }^{43}$. Theoretical nonadiabatic dynamics simulations employing the semiempirical OM2-multireference configuration interaction method combined with the QM/MM approach have yielded a lifetime of $410 \mathrm{fs}^{44}$, whereas in the framework of the timedependent density functional tight-binding method, explicitly including the water molecules in the first solvation shell, the nonadiabatic relaxation proceeds within less than $200 \mathrm{fs}^{45}$.

In the context of this ultrafast nonadiabatic relaxation, the aim of the present paper is to explore to which extent the excited state lifetimes of adenine can be affected using simple parameterized pulse trains, thus avoiding the need for full closed-loop field optimization. This represents the first step towards the manipulation of the excited state lifetimes of complex systems with the ultimate goal to selectively induce the fluorescence of DNA nucleobases.

The paper is structured as follows: In the Section 2, the theoretical approach is outlined by a brief presentation of the field-induced surface hopping method (FISH) as well as its combination with the quantum mechanical/molecular mechanical (QM/MM) approach and the procedure for the design of laser pulse trains. Subsequently, the computational details are given in Section 3. The results of the nonadiabatic dynamics of solvated adenine driven by laser pulse trains are presented in Section 4. Finally, conclusions and outlook are given in Section 5.

\section{Theoretical Approach}

We present here the extension of our FISH method ${ }^{31}$ by combining it with the quantum mechanical-molecular mechanical (QM/MM) approach which allows to treat the laser driven dynamics in complex systems interacting with various types of environments such as solvents, surfaces or proteins. Furthermore, we introduce here analytically parametrized laser pulse trains which will be subsequently applied for the manipulation of excited state dynamics of adenine in water. Although in this contribution we apply only analytically parametrized fields, we wish to emphasize that the combination of our approach with the optimal control theory is straightforward. We first briefly review the FISH method, then outline its combination with QM/MM and finally describe the design of laser pulse trains for the control of the excited state dynamics of solvated adenine.

\subsection{Field-induced surface hopping method (FISH)}

The idea of the FISH method is to propagate independent classical trajectories in a manifold of several electronic states and to describe the population transfer between the electronic states which arises both due to the coupling with the laser field 
as well as through nonadiabatic coupling by allowing the trajectories to switch between the states. This is similar to Tully's surface hopping method ${ }^{29}$ which is however only suited to describe field-free nonadiabatic transitions in molecular systems ${ }^{40,41,46-50}$.

The description of FISH simulations has been presented in detail in Ref. ${ }^{31,51}$. Briefly, the following steps are needed: First, initial conditions for an ensemble of trajectories have to be generated. Second, along each trajectory which is propagated in the framework of MD "on the fly" the electronic degrees of freedom are propagated by solving the timedependent Schrödinger equation in the manifold of adiabatic electronic states coupled by the laser field $\vec{E}(t)$ and by the nonadiabatic coupling terms $D_{i j}=\left\langle\psi_{i} \mid \frac{\partial \psi_{j}}{\partial t}\right\rangle$

$$
\begin{aligned}
i \hbar \dot{c}_{i}(t)= & E_{i}(\mathbf{R}(t)) c_{i}(t) \\
& -\sum_{j}\left[i \hbar D_{i j}(\mathbf{R}(t))+\vec{\mu}_{i j}(\mathbf{R}(t)) \cdot \vec{E}(t)\right] c_{j}(t),
\end{aligned}
$$

where $c_{i}(t)$ are the expansion coefficients of the electronic wavefunction from which the density matrix elements can be calculated as $\rho_{i j}=c_{i}^{*} c_{j}$. Finally, the hopping probabilities are determined in each nuclear time step from the change of the quantum electronic state populations $\rho_{i i}$ according to

$$
P_{i \rightarrow j}=\Theta\left(-\dot{\rho}_{i i}\right) \Theta\left(\dot{\rho}_{j j}\right) \frac{-\dot{\rho}_{i i}}{\rho_{i i}} \frac{\dot{\rho}_{j j}}{\sum_{k} \Theta\left(\dot{\rho}_{k k}\right) \dot{\rho}_{k k}} \Delta t
$$

where the $\Theta$ functions are defined to be one for positive arguments and zero otherwise. The hopping probabilities are used in a stochastic process to decide if a state switch occurs. In the absence of a laser field, the total energy of the system during a state switch is conserved by rescaling the component of the nuclear velocities in the direction of the nonadiabatic coupling vector. If a laser field is present, energy exchange with the molecular system can occur and thus no velocity rescaling is applied. The electronic state population as a function of time is finally obtained by averaging over the ensemble of trajectories.

The above presented FISH method is suitable for the simulation and control of laser-induced processes ${ }^{25,31}$ as well as for the simulation of spectroscopic observables such as timeresolved photoelectron spectra ${ }^{51-53}$ or harmonic emission ${ }^{54}$.

\subsection{Combination of FISH with the QM/MM approach}

Here we present the combination of the FISH method with QM/MM. This represents a general approach to perform nonadiabatic dynamics simulations under direct influence of laser fields for molecular systems interacting with their environment, thus extending our previous work on field-free QM/MM nonadiabatic dynamics in the framework of time-dependent density functional theory ${ }^{55}$. Due to the explicit inclusion of the laser field, also complex pulse forms suitable for manipulation and control of excited state dynamics can be employed. The basic idea of the QM/MM approach relies on a partition of the system in one part which is described fully quantum mechanically (QM part), and another part (the environment), for which a molecular mechanics treatment is sufficient (MM part). The interaction of the QM and MM subsystems can be accounted for by different approximations. In the mechanical embedding scheme, which has been used in the present contribution, only the steric repulsion between the QM and MM parts is considered, while the electronic structure of the QM system is not influenced by the MM part. In contrast, the electrostatic embedding scheme employed in Ref. ${ }^{55}$ takes into account the polarization of the QM wavefunction due to the environment by including the point charges from the MM part in the QM Hamiltonian. Since the electrostatic embedding is significantly more time consuming in the context of FISH simulations in which large number of control pulses are used to drive the dynamics of an ensemble of trajectories, we employ here the computationally more efficient mechanical embedding scheme. Moreover, the comparison between the mechanical and electrostatic embedding schemes on selected trajectories gave very similar results, thus giving us the confidence to use the mechanical embedding for large scale FISH simulations. In the mechanical embedding, if both the QM system (X) and the MM system (Y) are well described by classical force fields, the effect of the steric repulsion between both subsystems can be accounted for by the difference of the $\mathrm{MM}$ energies of the combined system $(\mathrm{X}+\mathrm{Y})$ and the QM system $(\mathrm{X})$ alone. The total energy of the combined system can then be written as

$$
E_{Q M / M M}=E_{Q M}(X)+E_{M M}(X+Y)-E_{M M}(X) .
$$

The forces needed to perform molecular dynamics "on the fly" are obtained as the gradient of Eq. (3), and both the nonadiabatic couplings as well as the transition dipole moments are calculated only for the QM part.

\subsection{Design of pulse trains for control of excited state dy- namics in solvated adenine}

In order to perform the control of the excited state dynamics we employ phase shaping by an analytical mask function in the spectral domain. Specifically, the laser field parameterized in the frequency domain as:

$$
E(\omega)=E_{0} \exp \left[-\frac{4 \ln 2}{w_{1 / 2}^{2}}\left(\omega-\omega_{0}\right)^{2}\right] \exp [i \phi(\omega)],
$$

with amplitude $E_{0}$, central frequency $\omega_{0}$, spectral width $w_{1 / 2}$ and phase $\phi$ has been modulated employing a sinusoidal phase 
modulation

$$
\phi(\omega)=a \sin \left[\tau\left(\omega-\omega_{0}\right)+c\right] .
$$

This procedure provides complex pulse forms in the time domain whose features are dependent only on the choice of the three parameters $a, \tau$, and $c$ and thus the control landscape can be fully sampled without the need for iterative optimization. In the time domain, the pulses defined by Eq. (4) consist of a train of temporally separated subpulses. The phase modulation parameters $a, \tau$, and $c$ determine the intensity variation within a pulse train, the time separation of the subpulses and the sine or cosine character of the modulation function, respectively.

\section{Computational Procedures}

The equilibrated structure of the solvated $9 \mathrm{H}$-adenine, which is the dominant species present in water, has been obtained by the following steps: First, adenine was placed into a cubic water box of $49.2 \AA$ side length, and classical force field molecular dynamics with periodic boundary conditions using the TINKER package ${ }^{56}$ was performed over 20 ps at a temperature of $300 \mathrm{~K}$ until the system was equilibrated. For adenine, the OPLS-AA force field parameters ${ }^{57-61}$ were used, while water was described by the TIP-3P parameters ${ }^{62}$. Subsequently, a water sphere with a radius of $35 \AA$ was cut around the adenine molecule, and the system was further equilibrated by performing a ground state $\mathrm{QM} / \mathrm{MM}$ dynamics simulation using the mechanical embedding scheme. The QM part consisted of the adenine molecule, for which the semiempirical OM2 method ${ }^{63,64}$ was employed. From the QM/MM trajectory obtained this way, a number of 100 initial conditions was finally generated by sampling at regular time intervals. In order to obtain an almost ideal water sphere, the water droplets of each structure were further truncated to a radius of $20 \AA$. In this way, adenine was solvated by about 1400 water molecules.

The FISH-QM/MM simulations have then been performed in the manifold of the ground and the three lowest excited states. For this purpose, the OM2 method combined with the GUGA multireference configuration interaction ${ }^{65,66}$ was employed in the framework of the MNDO program ${ }^{67}$, and all single and double excitations out of the four most relevant configurations of the considered electronic states were taken into account. The nuclear trajectories were propagated by numerical solution of Newton's equations of motion using the velocity Verlet algorithm ${ }^{68}$ with a time step of 0.25 fs. The total propagation time depended on the applied laser field and was chosen to be $\sim 1000$ fs longer than the duration of the field. Along each trajectory $\mathbf{R}(t)$, the time-dependent Schrödinger equation (1) including the nonadiabatic coupling and the coupling to the laser field was integrated using the Runge-Kutta

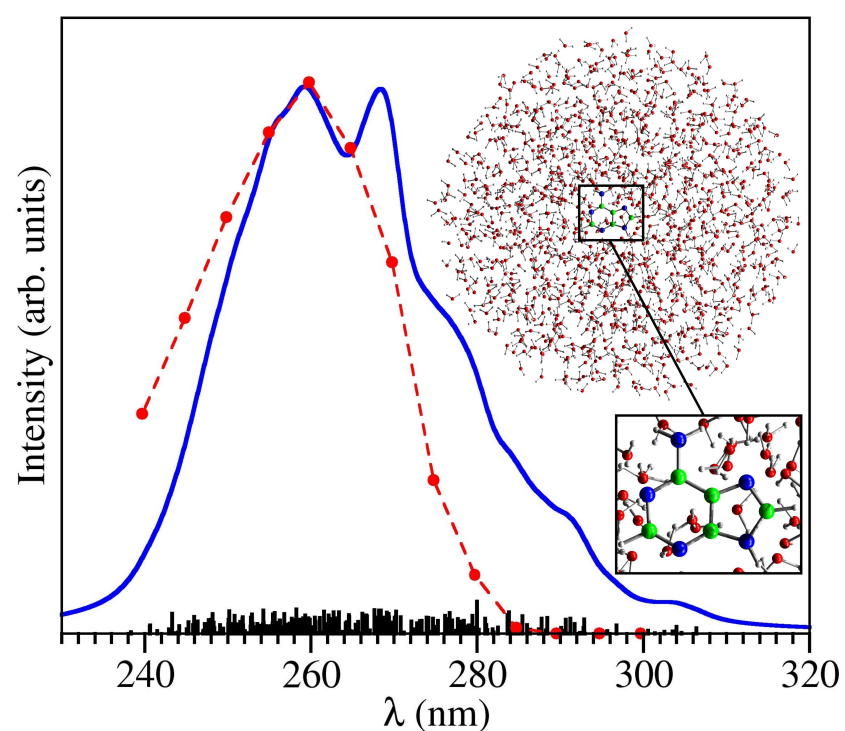

Fig. 1 Theoretical electronic absorption spectrum of adenine in a water sphere for the ensemble of 100 initial conditions. The individual transitions (black sticks) have been broadened by a Lorentzian width of $5 \mathrm{~nm}$ (blue curve). For comparison, the experimental absorption spectrum of Kohler et al. ${ }^{37}$ is also shown (red points).

procedure with a time step of $2.5 \cdot 10^{-5}$ fs. The electronic state coefficients obtained in this way were used to calculate the hopping probabilities according to Eq. (2). If the average absolute value of the field within a nuclear time step was lower than $10^{-6}$ a.u., the nuclear velocities were rescaled in the direction of the nonadiabatic coupling vector in order to assure energy conservation. For higher field strengths, energy exchange with the field was assumed and thus no velocity rescaling was applied.

The laser fields employed in the simulations were parameterized in the frequency domain using a sinusoidal phase modulation as described in Section 2.3. In our simulations, we have used 1024 discrete frequency values in the range between $4.33 \mathrm{eV}(287 \mathrm{~nm})$ and $4.98 \mathrm{eV}(249 \mathrm{~nm})$ around a central frequency of $4.66 \mathrm{eV}(266 \mathrm{~nm})$. The Gaussian width $w_{1 / 2}$ of the spectral amplitude was $0.19 \mathrm{eV}$. For the phase modulation parameters the values $a=\frac{\pi}{2}, \pi, \frac{3}{2} \pi, 2 \pi, \tau=20 \mathrm{fs}, 40$ fs, $60 \mathrm{fs}, 80 \mathrm{fs}, 100 \mathrm{fs}$ and $c=0, \frac{\pi}{2}, \pi, \frac{3}{2} \pi$ have been used, giving 80 different pulse trains in total. The pulses in the time domain are obtained by discrete Fourier transformation as $E(t)=\sum_{n} E\left(\omega_{n}\right) \exp \left(i \omega_{n} t\right)$. The unshaped pulse has a duration of $50 \mathrm{fs}$ (full width at half maximum of $20 \mathrm{fs}$ ), whereas the durations of the pulse trains vary between 150 and $2000 \mathrm{fs}$ depending on the pulse parameters. The maximum field amplitudes of the pulses correspond to intensities of $\sim 7 \cdot 10^{12} \frac{\mathrm{W}}{\mathrm{cm}^{2}}$. 


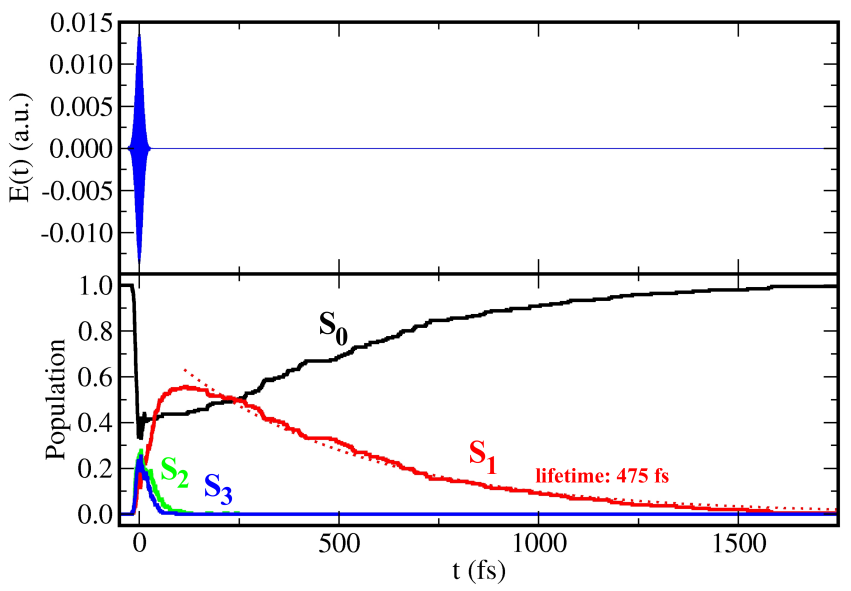

Fig. 2 Upper panel: Unshaped fourier-limited laser pulse at $266 \mathrm{~nm}$ for resonant excitation of adenine. Lower panel: Population dynamics of solvated adenine induced by the unshaped laser pulse, showing almost complete return to the ground state after $1000 \mathrm{fs}$.

\section{Results and Discussion}

In this section we present the results of the simulation of the excited state dynamics of solvated adenine under the influence of laser pulse trains obtained by the combination of FISH dynamics with the QM/MM approach as outlined in Section 3. We demonstrate that the pulse trains can be designed such that the course of the excited state dynamics is modified and the residence time of adenine in the electronically excited states is significantly extended.

The calculated electronic absorption spectrum of adenine embedded in a water sphere of $20 \AA$ radius is shown in Fig. 1 together with the experimental spectrum of Kohler et al. ${ }^{37}$. The maximal absorption is located between 250 and $280 \mathrm{~nm}$ for both the theoretical and the experimental spectrum, with a slight red shift of the theoretical spectrum. As it has been discussed in the literature, the electronic structure of adenine in this wavelength range is characterized by two bright states of $\pi \pi^{*}$ character $\left(L_{a}\right.$ and $\left.L_{b}\right)$ as well as by a low-lying dark $n \pi^{*}$ state $^{69-71}$. The exact ordering of these states has been found to strongly depend on the used quantum chemical method as well as on the actual structure for which the calculation is performed ${ }^{40,72-74}$. Therefore, within an ensemble of initial conditions necessary to simulate a temperature-broadened spectrum a decisive state ordering cannot be given and is of minor importance for the properties of the system.

Irradiation of solvated adenine by an ultrashort resonant laser pulse of $266 \mathrm{~nm}$ wavelength leads to simultaneous excitation of the three lowest excited states, as shown in Fig. 2. This is followed by ultrafast population transfer to the $S_{1}$ state which takes place within $\sim 50 \mathrm{fs}$. The population of this state

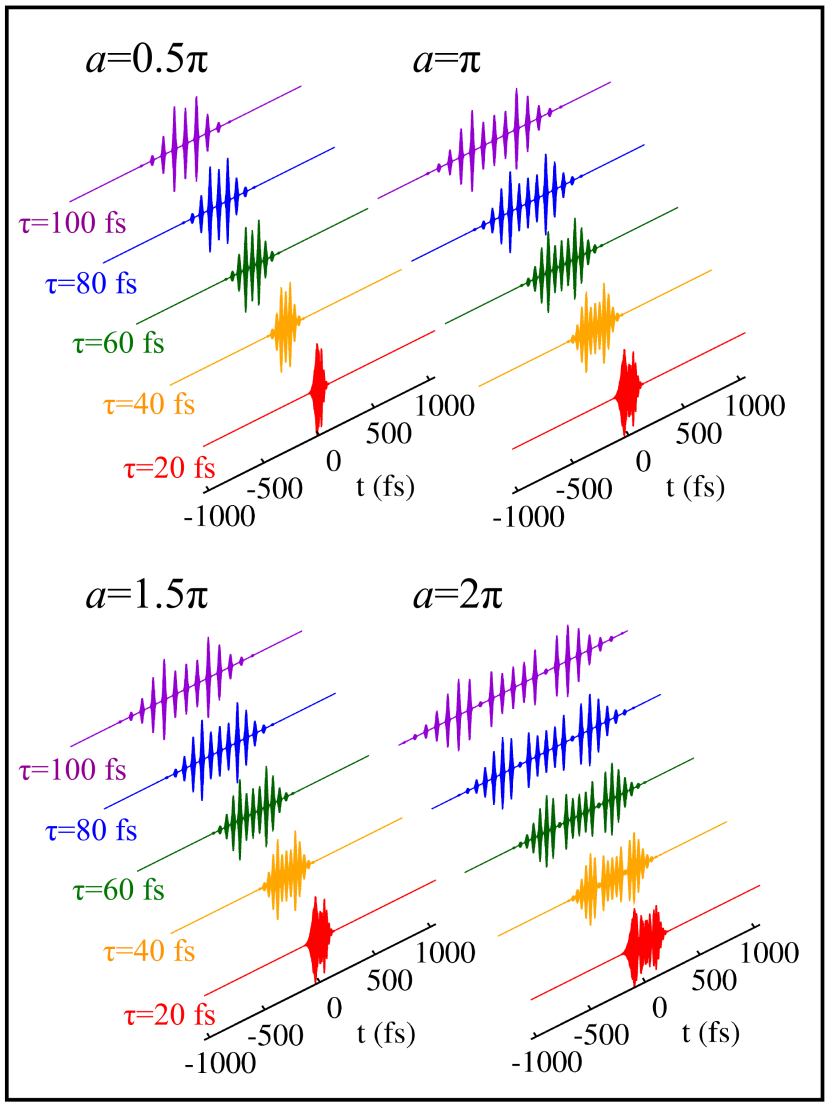

Fig. 3 Time profile of the pulse trains constructed by sinusoidal phase modulation $\phi(\omega)=a \sin \left(\tau\left(\omega-\omega_{0}\right)\right)$, employing values for $a$ between $0.5 \pi$ and $2 \pi$ and values for $\tau$ between 20 and $100 \mathrm{fs}$.
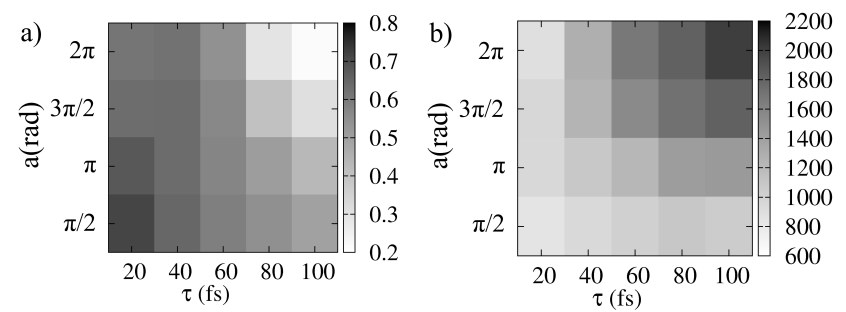

Fig. 4 Effect of the laser pulses shown in Fig. 3 on the excited state dynamics in adenine as function of $a$ and $\tau$. a) Maximal population of the $S_{1}$ state after the end of the pulse train. b) Time duration in fs until the $S_{1}$ population drops below $30 \%$ after the end of the pulse train. High values are indicated by dark, low values by light grey levels. 


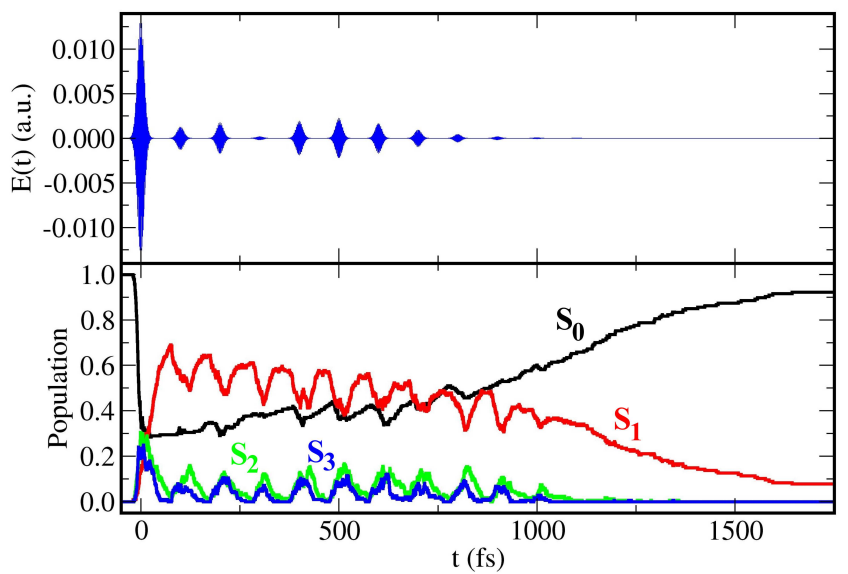

Fig. 5 Upper panel: Efficient pulse train obtained by superposition of the unshaped pulse and a pulse train with $a=2 \pi$ and $\tau=80 \mathrm{fs}$. Lower panel: Population dynamics induced by the laser pulse train which keeps the excited state population above $40 \%$ for $1000 \mathrm{fs}$.

reaches almost $60 \%$ and subsequently decays to the ground state with a time constant of $475 \mathrm{fs}$. This finding is comparable to the theoretical and experimental results discussed in the Introduction.

In order to investigate the influence of the shape of the laser pulse on the dynamical processes in the excited states, we constructed a set of pulse trains starting from the Fourier-limited Gaussian pulse in the frequency domain. For this purpose, the phase modulation by a sine function (cf. Eq. (5)) was applied and in total, 80 pulse trains have been generated by employing the parameter values given in Section 3. These pulse trains differ in the time delay of their subpulses as well as in the intensity pattern within the pulse train. For illustration, the time profiles of the pulse trains with parameter $c=0$ are shown in Fig. 3. The QM/MM FISH dynamics has been performed employing all 80 pulses with an ensemble of 50 trajectories, and the ability of the pulses to efficiently excite the adenine and to keep population in excited states has been assessed. The two-dimensional section out of the three-dimensional control landscape for $c=0$ is presented as a function of parameters $a$ and $\tau$ in Fig. 4. As a control target the maximal $\mathrm{S}_{1}$ population is presented in Fig. 4a. The largest population transfer to the $\mathrm{S}_{1}$ state is obtained for small values of $a$ and $\tau$. The associated temporal pulse form is characterized by a short pulse duration of $\sim 150$ fs and overlapping subpulses (cf. Fig. 3), thus only a small difference to an unshaped pulse is present. In order to reveal the influence of the pulse parameters on the time interval during which a significant population is kept in the excited states, we present in Fig. $4 \mathrm{~b}$ the time range in which the $S_{1}$ populations is kept above a threshold of $30 \%$. The longest time intervals are obtained for high values both of $a$ and $\tau$, which are not efficient for achieving a high population of the

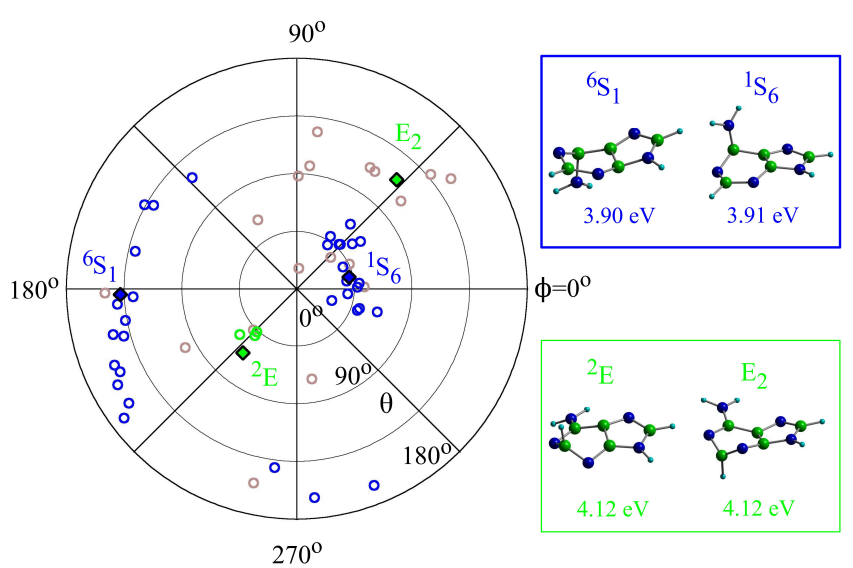

Fig. 6 Distribution of Cremer-Pople parameters for dynamics induced by the pulse train of Fig. 5 for the structures at final hops from $S_{1}$ to $S_{0}$. The structures of the lowest-lying conical intersections of adenine are shown on the right. The individual structures are classified according to their similarity to the structure of a conical intersection. Blue circles: close to ${ }^{1} \mathrm{~S}_{6} /{ }^{6} \mathrm{~S}_{1}$, green circles: close to ${ }^{2} \mathrm{E} / \mathrm{E}_{2}$, brown circles: structure not in the vicinity of a conical intersection.

$\mathrm{S}_{1}$ state. In the corresponding pulse trains, the total pulse duration amounts to 2 ps and the subpulses are well separated. Moreover, the total pulse intensity is distributed over a larger time interval, thus leading to lower field amplitudes for the individual subpulses (cf. Fig. 3). Very similar results are also found for the other values of parameter $c$.

Based on these findings, a laser pulse has been constructed with the aim to simultaneously achieve a high initial excitation efficiency and to keep the population in excited states for a sufficient time. For this purpose the original unshaped pulse was superposed by the pulse train obtained with the parameter values $a=2 \pi, \tau=80$ fs and $c=0$. The temporal structure of the resulting pulse beginning at $\mathrm{t}=0 \mathrm{fs}$ is shown in Fig. 5 . The population dynamics induced by this pulse, also shown in Fig. 5, is distinctly different to that due to the unshaped pulse (cf. Fig. 2). Initial excitation to all three excited states by the first intensive subpulse is followed by ultrafast decay of the $S_{2}$ and $S_{3}$ population to the $S_{1}$ within $50 \mathrm{fs}$. However, before further relaxation to the ground state can occur, the next subpulse leads to re-excitation from $S_{1}$ to $S_{2}$ and $S_{3}$. In this way, the population is cycled between the $S_{1}$ and higher $S_{2}$ and $S_{3}$ states by a series of pump excitations followed by nonadiabatic decay to the $S_{1}$ state. These pump excitations induced by the subpulses of the train serve to prevent the nonadiabatic relaxation of the $S_{1}$ state to the ground state which would naturally occure without the control pulse. In this way the population is kept in the excited states for about $1000 \mathrm{fs}$, which is significantly longer than the intrinsic lifetime of $500 \mathrm{fs}$. The 


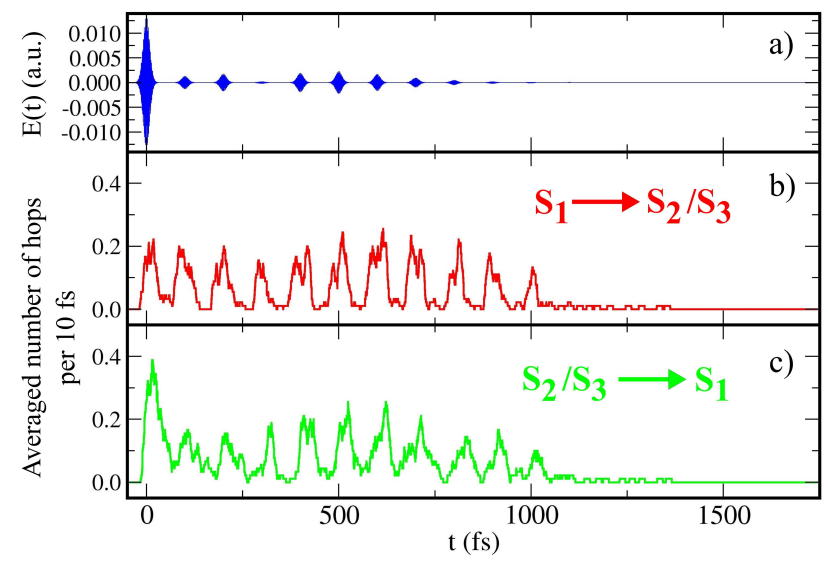

Fig. 7 Time-averaged distribution of hopping events from the $S_{1}$ up to the $S_{2}$ and $S_{3}$ states (b) and back (c) for the dynamics induced by the pulse train of Fig. 5 (a). The subpulses of the train consistently repopulate the higher $S_{2}$ and $S_{3}$ states and thus decrease the time spent in $\mathrm{S}_{1}$ where the trajectories would quickly move towards a conical intersection.

dynamical processes under the influence of the pulse train as well as of the unshaped pulse are characterized by motions involving the lowest-frequency modes in which distortions of the ring system take place.

From the population dynamics shown in Fig. 5 it becomes clear that after the pulse train has ceased, nonadiabatic relaxation to the ground state occurs within about $500 \mathrm{fs}$, which is similar to the relaxation induced by an unshaped pulse (cf. Fig. 2). After pulse has ceased, in most cases the ground state is reached at a very small $S_{1}-S_{0}$ energy gap, indicating that the transition happens in the vicinity of a conical intersection (CI). The characterization of CIs in ring systems can be accomplished by classifying their structure according to the Cremer Pople parameters ${ }^{75}$, which quantify the ring puckering for a given CI. In the case of adenine, there are two doubly degenerate CIs relevant for the nonadiabatic dynamics ${ }^{40,41}$, which are classified as ${ }^{1} \mathrm{~S}_{6} /{ }^{6} \mathrm{~S}_{1}$ and ${ }^{2} \mathrm{E} / \mathrm{E}_{2}$ in the notation introduced by Boeyens ${ }^{76}$. The ${ }^{1} \mathrm{~S}_{6} /{ }^{6} \mathrm{~S}_{1} \mathrm{CI}$ has the lowest energy $(3.9 \mathrm{eV}$ above the ground state minimum), whereas the ${ }^{2} \mathrm{E} / \mathrm{E}_{2} \mathrm{CI}$ lies about $0.2 \mathrm{eV}$ higher. As has been recently found by Thiel et al., within the OM2 approach the nonadiabatic relaxation of adenine to the ground state proceeds mostly in the vicinity of the ${ }^{1} \mathrm{~S}_{6} /{ }^{6} \mathrm{~S}_{1} \mathrm{CI}^{44}$ both in the gas phase and in water. In order to investigate how formed pulse trains influence the dynamical pathways taken in the excited electronic states, the Cremer Pople parameters for the final hops from $S_{1}$ to $S_{0}$ have been determined for the trajectories driven by the pulse train of Fig. 5 and are shown in Fig. 6. Evidently, most of the trajectories hop in the vicinity of the ${ }^{1} \mathrm{~S}_{6} /{ }^{6} \mathrm{~S}_{1}$ CIs, similar to the case of field-free nonadiabatic dynamics while the contribution of

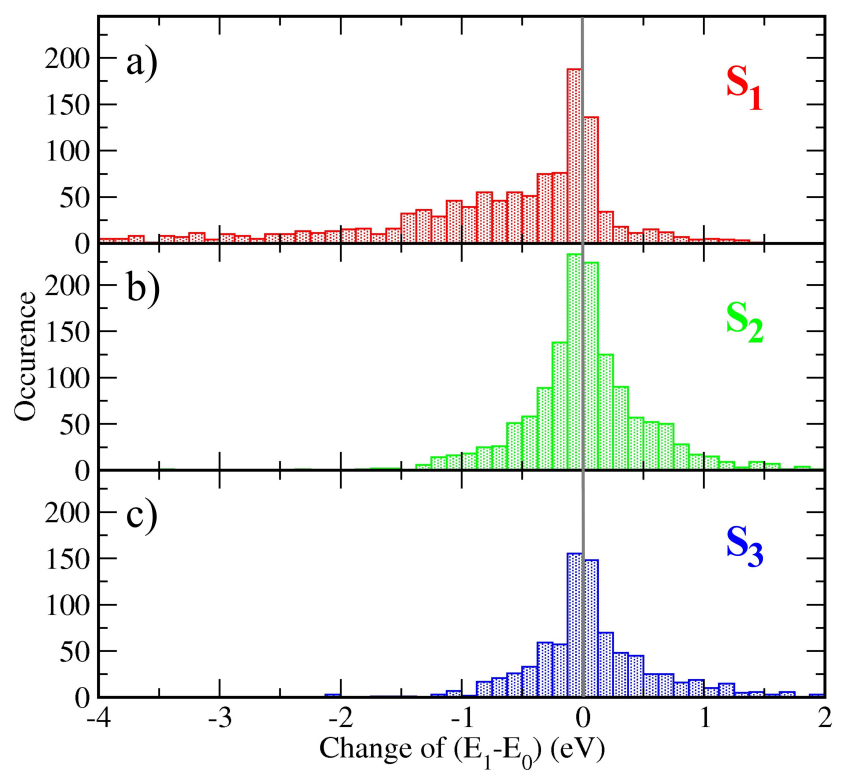

Fig. 8 Change of the energy gap between $S_{0}$ and $S_{1}$ state for all time intervals when a trajectory resides in the states $S_{1}(a), S_{2}(b)$ or $\mathrm{S}_{3}$ (c) for the dynamics induced by the pulse train of Fig. 5. For the $\mathrm{S}_{1}$ state the average value is negative $(-0.7 \mathrm{eV})$, indicating straightforward motion towards a conical intersection. In contrast, the average value is slightly positive for the $S_{2}(+0.02 \mathrm{eV})$ and $S_{3}$ $(+0.1 \mathrm{eV})$ states, which means that the trajectories depart from the vicinity of conical intersections.

the ${ }^{2} \mathrm{E} / \mathrm{E}_{2} \mathrm{CI}$ is negligible. The trajectories driven by the unshaped Gaussian pulse behave also similarly. Thus, the relaxation mechanism to the ground state is the same regardless if the excitation is achieved using the unshaped Gaussian pulse or a pulse train. However, the excited state population dynamics while the pulses are acting is fundamentally different. As discussed above, the dynamics induced by the pulse train is characterized by a sequence of excitations and de-excitations between the $S_{1}$ state and the higher-lying $S_{2}$ and $S_{3}$ states. This can be quantified by analyzing the hopping events between these states as a function of time as shown in Fig. 7. The highest number of hops between the states coincides with the presence of a subpulse. Immediately before a subpulse acts, almost the complete excited state population resides in $\mathrm{S}_{1}$ (cf. Fig. 5). Therefore, first the hops from there up to $\mathrm{S}_{2} / \mathrm{S}_{3}$ occur and reach their maximal number shortly before the highest field amplitude of the subpulse (cf. Fig. 7b). With increasing occupation of $S_{2}$ and $S_{3}$, also hops back to $S_{1}$ are induced (cf. Fig. 7c). The maximal number of such events is reached a short time after the field maxima. When the subpulse ceases, the nonadiabatic coupling between the excited states still invokes hops from $S_{2}$ and $S_{3}$ down to $S_{1}$, leading to rapid depopulation of these higher states (cf. Figs. 5 and $7 \mathrm{c}$ ). 
In contrast to this behaviour, the unshaped pulse only once excites the molecules followed by a gradual relaxation from the bright states to the $S_{1}$ state, and further to the ground state (cf. Fig. 2).

Therefore, in average, a trajectory driven by the pulse train resides much longer in the higher excited states, which thus influence significantly more the dynamics than in the case of the unshaped pulse. Since the efficiency of the nonadiabatic relaxation to the ground state is related to the ability to reach a $C I$ between the $S_{1}$ and $S_{0}$ states, the temporal change of the $\mathrm{S}_{1}-\mathrm{S}_{0}$ energy gap can be used to monitor if a given trajectory moves towards a CI or not. This analysis has been performed for the trajectories driven by the pulse train for all time periods during which they resided in one of the excited states and is shown in Fig. 8. It becomes evident that when a trajectory is propagated in $S_{1}$, in most cases the energy gap to the ground state becomes smaller (in average, by $-0.7 \mathrm{eV}$ ), indicating that the dynamics is straightforwardly directed towards a CI. In contrast, the dynamics in $\mathrm{S}_{2}$ and $\mathrm{S}_{3}$ can lead both to an increase or to a decrease of the energy gap, with the tendency towards the increase, as the average change is $+0.02 \mathrm{eV}$ for $\mathrm{S}_{2}$ and $+0.1 \mathrm{eV}$ for $\mathrm{S}_{3}$. This means that reaching nuclear configurations in the vicinity of an $\mathrm{S}_{0} / \mathrm{S}_{1} \mathrm{CI}$ is the more impeded the longer a trajectory is propagated in $S_{2}$ or $S_{3}$ states, and therefore the excited state lifetime can be elongated when these states are occupied more frequently. To achieve this, it is not necessary to continuously irradiate the system. Instead, we have shown here that a pulse train with temporally well separated weak features is sufficient to keep the population in excited states. Thus the main effect of this pulse train is to exploit the different shapes of the $\mathrm{S}_{2}$ and $\mathrm{S}_{3}$ potential energy surfaces, thus hindering the direct approach to the CI geometries, which helps prevent the decay to the ground state for a longer time.

\section{Conclusions}

We have demonstrated how appropriately designed simple laser pulse trains can be used to manipulate the excited state dynamics of adenine in water to elongate the excited state lifetime. For this purpose, we have introduced the combination of the FISH method with the QM/MM approach in order to include the water environment in the laser driven dynamics simulations. Utilizing analytical phase modulation in the frequency domain with a limited set of parameters, we have constructed a pulse train which after initial excitation can keep the excited states in adenine populated for a longer time. This occurs by sequential re-excitation from the first into higher excited states, competing with the nonradiative ultrafast decay back to the first excited state. In this way, the landscape of higher potential energy surfaces in which the access to structures of conical intersections is hindered can be exploited in order to prevent fast decay to the ground state. Such elongation of the excited state population could represent a means for increasing the fluorescence yield in molecules that intrinsically exhibit ultrafast nonradiative relaxation. This is of large interest since the enhancement of fluorescence is an important issue in the context of applications for biosensing.

\section{Acknowledgement}

We acknowledge financial support by the Deutsche Forschungsgemeinschaft, FOR 1282 (J. P. and R. M.), SPP 1391 (R.M. and V.B.-K.) and Emmy-Noether Program (MI-1236) (R. M. and M. W.). In addition, H. L. thanks the Austrian Science Fund, Special Research Program and F41 Vienna Computational Materials Laboratory (ViCoM) as well as the Robert A. Welch Foundation (Grant No. D-0005) for financial support.

\section{References}

1 D. J. Tannor and S. A. Rice, J. Chem. Phys., 1985, 83, 5013.

2 D. J. Tannor and S. A. Rice, Adv. Chem. Phys., 1988, 70, 441.

3 P. Brumer and M. Shapiro, Faraday Discuss. Chem. Soc., 1986, 82, 177.

4 M. Shapiro and P. Brumer, J. Chem. Phys., 1986, 84, 4103.

5 M. Shapiro and P. Brumer, Int. Rev. Phys. Chem., 1994, 13, 187.

6 A. M. Weiner, Rev. Sci. Instrum., 2000, 71, 1929.

7 S. A. Rice and M. Zhao, Optical Control of Molecular Dynamics, John Wiley\& Sons, Inc., New York, 2000.

8 P. W. Brumer and M. Shapiro, Principles of the Quantum Control of Molecular Processes, Wiley-VCH, Berlin, 2003.

9 M. Dantus and V. V. Lozovoy, Chem. Rev., 2004, 104, 1813.

10 Analysis and Control of Ultrafast Photoinduced Reactions, ed. L. Wöste and O. Kühn, Springer Series in Chemical Physics 87, 2007.

11 A. Baltuska, T. Udem, M. Uiberacker, M. Hentschel, E. Goulielmakis, C. Gohle, R. Holzwarth, V. S. Yakovlev, A. Scrinzi, T. W. Hänsch and F. Krausz, Nature, 2003, 421, 611.

12 E. Goulielmakis, V. S. Yakovlev, A. L. Cavalieri, M. Uiberacker, V. Pervak, A. Apolonski, R. Kienberger, U. Kleineberg and F. Krausz, Science, 2007, 317, 769.

13 C. Winterfeldt, C. Spielmann and G. Gerber, Rev. Mod. Phys., 2008, 80, 117.

14 M. Wollenhaupt and T. Baumert, Faraday Discuss., 2011, 153, 9.

15 T. Brixner and G. Gerber, ChemPhysChem, 2003, 4, 418.

16 W. Wohlleben, T. Buckup, J. L. Herek and M. Motzkus, ChemPhysChem, 2005, 6, 850 .

17 P. Nuernberger, D. Wolpert, H. Weiss and G. Gerber, Proc. Nat. Acad. Sc. USA, 2010, 107, 10366.

18 R. S. Judson and H. Rabitz, Phys. Rev. Lett., 1992, 62, 1500.

19 A. Assion, T. Baumert, M. Bergt, T. Brixner, B. Kiefer, V. Seyfried, M. Strehle and G. Gerber, Science, 1998, 282, 919.

20 C. Daniel, J. Full, L. Gonzalez, C. Lupulescu, J. Manz, A. Merli, S. Vajda and L. Wöste, Science, 2003, 299, 536.

21 S. Vajda, A. Bartelt, E.-C. Kaposta, T. Leisner, C. Lupulescu, S. Minemoto, P. Rosenda-Francisco and L. Wöste, Chem. Phys., 2001, 267, 231.

22 B. Schäfer-Bung, R. Mitrić, V. Bonačić-Koutecký, A. Bartelt, C. Lupulescu, A. Lindinger, S. Vajda, S. M. Weber and L. Wöste, J. Phys. Chem. A, 2004, 108, 4175 . 
23 V. Bonačić-Koutecký and R. Mitrić, Chem. Rev., 2005, 105, 11.

24 G. Vogt, G. Krampert, P. Niklaus, P. Nuernberger and G. Gerber, Phys. Rev. Lett., 2005, 94, 068305.

25 J. Petersen, R. Mitrić, V. Bonačić-Koutecký, J.-P. Wolf, J. Roslund and H. Rabitz, Phys. Rev. Lett., 2010, 105, 073003.

26 A. Bartelt, A. Lindinger, C. Lupulescu, S. Vajda and L. Wöste, Phys. Chem. Chem. Phys., 2003, 5, 3610.

27 A. F. Bartelt, T. Feurer and L. Wöste, Chem. Phys., 2005, 318, 207.

28 G. Vogt, P. Nuernberger, R. Selle, F. Dimler, T. Brixner and G. Gerber, Phys. Rev. A, 2006, 74, 033413.

29 J. C. Tully, J. Chem. Phys., 1990, 93, 1061.

30 J. C. Tully, Faraday Discuss., 1998, 110, 407.

31 R. Mitrić, J. Petersen and V. Bonačić-Koutecký, Phys. Rev. A, 2009, 79, 053416.

32 P. Nuernberger, G. Vogt, T. Brixner and G. Gerber, Phys. Chem. Chem. Phys., 2007, 9, 2470.

33 A. Warshel and M. Levitt, J. Mol. Biol., 1976, 103, 227.

34 M. J. Field, P. A. Bash and M. Karplus, J. Comput. Chem., 1990, 11, 700

35 J. Gao, in Reviews in Computational Chemistry, ed. K. B. Lipkowski and D. B. Boyd, Wiley, New York, 1995, vol. 7, p. 119.

36 H. M. Senn and W. Thiel, in Atomistic Approaches in Modern Biology, ed. M. Reiher, Topics Current Chemistry, 2007, vol. 268, p. 173.

37 B. Cohen, P. M. Hare and B. Kohler, J. Am. Chem. Soc., 2003, 125, 13594

38 S. Ullrich, T. Schultz, M. Z. Zgierski and A. Stolow, J. Am. Chem. Soc., 2004, 126, 2262.

39 D. C. Lührs, J. Viallon and I. Fischer, Phys. Chem. Chem. Phys., 2001, 3, 1827.

40 E. Fabiano and W. Thiel, J. Phys. Chem. A, 2008, 112, 6859.

41 M. Barbatti and H. Lischka, J. Am. Chem. Soc., 2008, 130, 6831.

42 T. Pancur, N. K. Schwalb, F. Renth and F. Temps, Chem. Phys., 2005, 313, 199.

43 B. A. West, J. M. Womick and A. M. Moran, J. Phys. Chem. A, 2011, 115, 8630.

44 Z. Lan, Y. Lu, E. Fabiano and W. Thiel, ChemPhysChem., 2011, 12, 1989.

45 R. Mitrić, U. Werner, M. Wohlgemuth, G. Seifert and V. BonačićKoutecký, J. Phys. Chem. A, 2009, 113, 12700.

46 R. Mitrić, V. Bonačić-Koutecký, J. Pittner and H. Lischka, J. Chem. Phys., 2006, 125, 024303

47 E. Tapavicza, I. Tavernelli and U. Rothlisberger, Phys. Rev. Lett., 2007, 98, 023001.

48 M. Barbatti, G. Granucci, M. Persico, M. Ruckenbauer, M. Vazdar, M. Eckert-Maksić and H. Lischka, J. Photochem. Photobiol. A.: Chem., 2007, 190, 228.
49 Z. Lan, E. Fabiano and W. Thiel, J. Phys. Chem. B, 2009, 113, 3548.

50 M. Vazdar, M. Eckert-Maksić, M. Barbatti and H. Lischka, Mol. Phys., 2009, 107, 845.

51 R. Mitrić, J. Petersen, M. Wohlgemuth, U. Werner, V. Bonačić-Koutecký, L. Wöste and J. Jortner, J. Phys. Chem. A, 2011, 115, 3755.

52 R. Mitrić, J. Petersen, M. Wohlgemuth, U. Werner and V. BonačićKoutecký, Phys. Chem. Chem. Phys., 2011, 13, 8690.

53 J. Stanzel, M. Neeb, W. Eberhardt, P. G. Lisinetskaya, J. Petersen and R. Mitrić, Phys. Rev. A, 2012, 85, 013201.

54 P. G. Lisinetskaya and R. Mitrić, Phys. Rev. A, 2011, 83, 033408.

55 M. Wohlgemuth, R. Mitrić and V. Bonačić-Koutecký, J. Chem. Phys., 2011, 135, 054105.

56 J. W. Ponder and F. M. Richards, J. Comput. Chem., 1987, 8, 1061.

57 W. L. Jorgensen, D. S. Maxwell and J. Tirado-Rives, J. Am. Chem. Soc., 1996, 117, 11225.

58 W. L. Jorgensen and N. A. McDonald, J. Mol. Struct.: THEOCHEM, 1998, 424, 145.

59 N. A. McDonald and W. L. Jorgensen, J. Phys. Chem. B, 1998, 102, 8049.

60 R. C. Rizzo and W. L. Jorgensen, J. Am. Chem. Soc., 1999, 121, 4827.

61 W. L. Jorgensen, J. P. Ulmschneider and J. Tirado-Rives, J. Phys. Chem. $B, 2004, \mathbf{1 0 8}, 16264$.

62 W. L. Jorgensen, J. Chandrasekhar, J. D. Madura, R. W. Impey and M. L. Klein, J. Chem. Phys., 1983, 79, 926.

63 W. Weber, PhD thesis, Universität Zürich (Switzerland), 1996.

64 W. Weber and W. Thiel, Theor. Chem. Acc., 2000, 103, 495.

65 A. Koslowski, M. E. Beck and W. Thiel, J. Comput. Chem., 2003, 24, 714.

66 S. Patchkovskii, A. Koslowski and W. Thiel, Theor. Chem. Acc., 2005, 114, 84.

67 W. Thiel, MNDO program, Max-Planck-Institut für Kohlenforschung, Mühlheim, Germany, 2007.

68 L. Verlet, Phys. Rev., 1967, 159, 98.

69 L. B. Clark and I. Tinoco, J. Am. Chem. Soc., 1965, 87, 11.

70 W. Voelter, R. Records, E. Bunnenberg and C. Djerassi, J. Am. Chem. Soc., 1968, 90, 6163.

71 A. Fucaloro and L. S. Forter, J. Am. Chem. Soc., 1971, 93, 1378.

72 C. M. Marian, J. Chem. Phys., 2005, 122, 104314.

73 L. Serrano-Andres, M. Merchan and A. Borin, Proc. Natl. Acad. Sci., 2006, 103, 8691.

74 T. Fleig, S. Knecht and C. Hättig, J. Phys. Chem. A, 2007, 111, 5491.

75 D. Cremer and J. A. Pople, J. Am. Chem. Soc., 1975, 97, 1354.

76 J. C. A. Boeyens, J. Chem. Crystallogr., 1978, 8, 317. 